\title{
EPJH Editorial
}

\author{
James D. Wells ${ }^{1, a}$ and Michael Eckert ${ }^{2, b}$ \\ 1 Physics Department, University of Michigan, Ann Arbor, USA \\ 2 Forschungsinstitut, Deutsches Museum, Munich, Germany
}

Published online 18 June 2021

(C) The Author(s), under exclusive licence to EDP Sciences, Società Italiana di Fisica and Springer-Verlag GmbH Germany, part of Springer Nature 2021, corrected publication 2021

We are honored to be the new Editors in Chief of European Physical Journal H. The journal has been led expertly since its founding by Wolf Beiglböck. The tradition of excellence that Prof. Beiglböck has given to this journal is certainly our first priority and the priority of the other Editors at EPJH.

We would like to take this opportunity to reiterate the traditional aims and scopes of EPJH, to explain how we wish to expand somewhat on this mission and conclude with an invitation to researchers to join the reading community of EPJH if they have not done so yet and to publish in the journal. This invitation applies not only to professional historians of science but also to those actively working in disciplinary physics who engage with history of science from time to time. It was and is our particular concern to establish for both with EPJH a common forum which is not provided by other journals in the same way.

First, as we state in our Aims and Scope page "The purpose of this journal is to catalyse, foster, and disseminate an awareness and understanding of the historical development of ideas in contemporary physics, and more generally, ideas about 'how Nature works'." And it should be emphasized that our journal exists to serve primarily the physics community. As such, many of the articles by historians of science are written expressly for an audience of practicing physicists.

Furthermore, there is a large number of articles written by active physicists themselves with the goal of elucidating the historical context of our present understanding in physics, or elucidating the path by which physics discoveries are made. These serve the physics community by enabling deeper understanding of the current physics landscape of knowledge, and helping us better understand the types of activities that pay off in physics research versus those that do not, which enlightens our own research programs.

One of the areas that we would like to expand in the pages of this journal is the role of "big science" in scientific discovery. There are other categories in the historical development of recent physics (inter- and transdisciplinarity, computational possibilities, etc.) that deserve attention and for which EPJH was and is open, but let us focus on "big science" in order to expose our intentions more clearly. What comes to mind first is particle physics. Of course, there has always been an element of big science in the discoveries of particle physics over the last few decades. Large collaborations of hundreds and even thousands of physicists have led to the discoveries of the $\mathrm{W}$ and $\mathrm{Z}$ bosons, the top quark and most recently the Higgs boson. These discoveries have been well documented, and the role of big science and big collaboration has been well appreciated within that subfield.

But other subfields beyond particle physics are increasingly being seen as "big science" endeavors. The recent discovery of gravity waves is a good example. This is a field that did not exist before, except for a few small-scale attempts over the last 50 years. Nevertheless, it was understood by a few scientists that a massive effort was needed to reach a qualitatively new level of understanding of gravity through direct detection of gravity waves. The benefit of this discovery is not just to confirm the existence of gravity waves, which was hardly a controversial topic, but rather the opening of an entirely new field of multi-messenger astronomy, which can see the effects of guaranteed phenomena, such as inspiraling black hole mergers, as well as probe more speculative ideas such as first-order phase transitions in the early universe or gravitational radiation from cosmic strings. The scientific richness is just beginning to be explored. There is an equally compelling historical story of how our understanding of gravity waves has progressed over the years, and our understanding of how they might be discovered. The pitfalls and the triumphs in this journey make for not only a compelling narrative but a scientifically enlightening story for researchers who may be faced with similar challenges as they develop the new forefront of discovery.

\footnotetext{
a e-mail: jwells@umich.edu (corresponding author)

be-mail: m.eckert@deutsches-museum.de
} 
The Quantum Science initiatives that are being created throughout the world are another element of big science that is defined less by "one big machine" but rather by "one big goal" — a workable quantum computer-with huge numbers of people and resources devoted to it. What are the challenges being faced by this unique large-scale effort? Do they overlap with the challenges CERN had in constructing the LHC with its primary goal of finding the Higgs boson, or LIGO with its goal of finding gravity waves? Does the existence of a central location (CERN or LIGO labs) for more or less everybody make a difference to how progress is made or how it can be tracked?

Significant parts of condensed matter physics are also entering the realm of big science. Materials research is now regularly being done at multi-billion-dollar facilities, such as high-flux research nuclear reactors, advanced light sources, and the neutron spallation sources that are being built (ESS in Sweden, for example) and upgraded (SNS in Oak Ridge, USA). How is the science changing? What challenges does the field face going from basement at the University to a central Lab in Lund, Sweden? Why did it become necessary to do that? Are prospects dwindling for big breakthroughs from small experiments, even in condensed matter physics, and if so, why?

These are some of the many questions that the era of big science is bringing to physics, and EPJH has plans to be on the forefront of these questions. For that reason, we will be inviting researchers in some of these emerging big science disciplines to contribute articles on the shifts this creates in how science is done, both in its disciplinary science aspects and the aspects associated with public policy. Increasingly, the motivation for doing science is a complex interaction between scientific justification and large-scale government investment. The scientists involved have perspectives that need to be heard in this area.

Finally, we are very pleased to be Editors at EPJH. Along with our readers and contributors, we deeply value the contributions that historical analysis and reflections can have for both understanding better what we think we already know in physics and leading us on better paths toward future understanding. We welcome your continuing involvement in this important scholarly endeavor. 